\title{
An efficient, inexpensive food hopper for monitoring feeding habits of rats
}

\author{
ALLEN D. BOSTWICK and JOHN J. PORTER \\ University of Wisconsin, Milwaukee, Wisconsin 53201
}

An efficient food hopper designed for the constant monitoring of feeding patterns of rats is described. The apparatus is inexpensive and is designed to attach to standard rat housing cages.

Conventional means for monitoring feeding patterns in rats, and rodents in general, have required either expensive collateral apparatus or time-consuming monitoring procedures. For example, the use of operant chambers, in which subjects may barpress for ad-lib food, requires substantial relay apparatus for operation and data collection, and studies are restricted to a small number of concurrent subjects due to the limited number of chambers available (cf. Bolles \& Stokes, 1965). The use of contact sensors (Bernstein, 1976) alleviates the problem of apparatus restrictions and enables continual monitoring of free-feeding eating patterns, but does not circumvent the necessity for expensive collateral apparatus. Finally, if animals are observed and their food consumption measured, the need for expensive apparatus is eliminated, but such methods are time-consuming and provide a relatively molar analysis of feeding (Bolles, 1963; Moll, 1964).

We have recently designed an efficient and inexpensive food hopper that provides assessment of continuous home-cage free-feeding eating patterns. The hopper is reliable, simple to construct, and serves as an excellent food hopper "postexperimentally."

The food hopper is constructed of approximately $442.0 \mathrm{sq} \mathrm{cm}$ of Plexiglas $(.6 \mathrm{~cm}$ thickness has provided a very durable and lightweight food hopper), approximately $80.0 \mathrm{sq} \mathrm{cm}$ of $.04-\mathrm{cm}$ sheet aluminum, and two conventional Nu-way snap studs. Spring-steel wire (16 gauge) is used for the swing-door axle and 32-gauge flexible wire is used for the power contact circuit. (The flexibility of the wire is critical in order to provide the least amount of resistance to the operation of the swinging door.) The Plexiglas may be joined with epoxy glue or any other stable adhesive. In addition, holes drilled in the floor of the food reservoir prevent food dust from collecting in the reservoir over time.

A perspective drawing of the intact food hopper is shown in Figure 1, Detail A. Detail B shows the angle of

The authors wish to acknowledge the contribution of Charles Hanson to the final design and assembly of the food hoppers. Requests for reprints should be sent to Allen D. Bostwick, Department of Psychology, University of Wisconsin, Milwaukee, Wisconsin 53201.
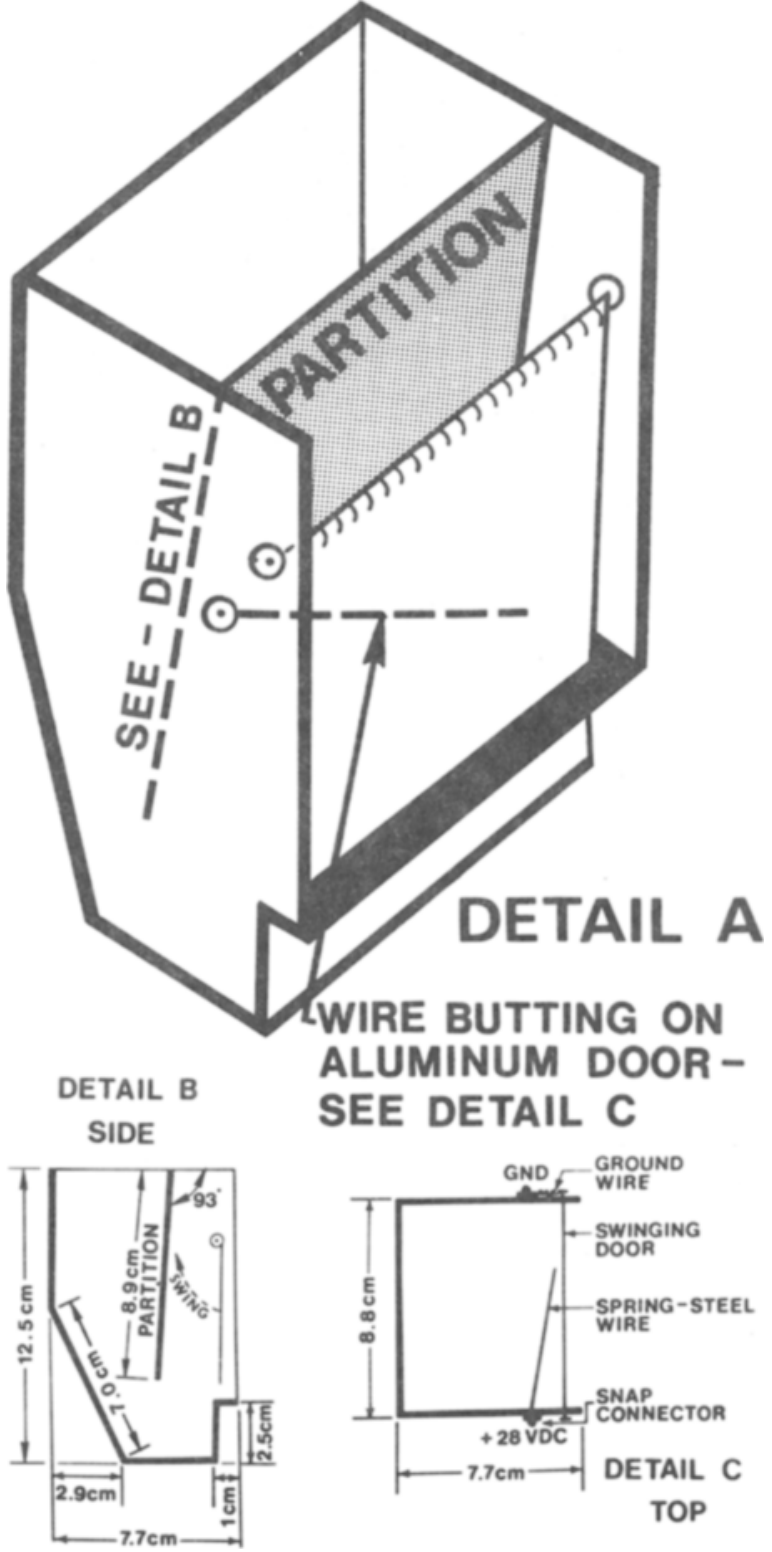

Figure 1. A perspective drawing of the intact food hopper is shown in Detail A. Detail B shows the position of the foodhopper partition and Detail $C$ shows the positioning of the spring-steel wire used to make circuit closure. 
incline of the hopper partition and the length of vertical extension into the hopper. Both of these dimensions are important for proper operation. While the partition serves to channel food into the animal's eating reservoir, a change in its angle either prohibits food entry into the reservoir or, conversely, allows the reservoir to fill to a level that may restrict movement of the swinging door. Detail $\mathrm{C}$ shows the most reliable means for positioning the spring-steel wire used to make circuit closure. Resistance to movement of the swinging door is minimized by extending the wire from one side of the food hopper to the other. The angle of the wire maximizes sensitivity to movement of the swinging door.

Some home-cage modification is required to install the hoppers. A $4 \times 4 \mathrm{~cm}$ opening must be cut in the front wall in order to permit the animal access to the swinging door and to the food reservoir itself.

The unit is designed to accommodate standard Purina-type rat chow pellets broken into quarters. This size pellet falls through the grid floor, which prevents possible hoarding behavior.

Although we used an Esterline-Angus Model (AW) 20-pen event recorder for data collection, other instruments can be used also. In addition to accommodating
20 subjects simultaneously, a paper speed of $25-50 \mathrm{~mm} / \mathrm{h}$ provides adequate sensitivity to "runs" in feeding behavior over time.

The hoppers have been tested over several continuous days and have proved trouble free. After a 2- to 3-day adaptation period, animals consume approximately the same quantity of food in the recording food hoppers as in the conventional food hopper attached to the animals' home cages.

\section{REFERENCES}

Bernstein, 1. L. Ontogeny of meal patterns of the rat. Journal of Comparative and Phisiological Psichology. 1976, 90. 1126-1132.

Bolles. R. C. Effect of food deprivation upon the rat's behavior in its home-cage. Joumal of Comparative and Physiological Psychology. 1963, 56. 456-460.

Bolles, R. C.. \& Stokes, L. W. Rat's anticipation of diurnal and a-diurnal feeding. Journal of Comparative and Physiological Psychology. 1965. 60, 290-294.

MoLl. R. P. Drive and maturation effects in the development of consummatory behavior. Psychological Reports, 1964. 15. 295-302.

(Received for publication June 1. 1977; revision accepted July 19.1977.) 